\title{
Smart Book Reader for Visual Impairment Person using IoT Device
}

\author{
Norharyati binti Harum* ${ }^{1}$, Nurul Azma Zakaria ${ }^{2}$, Nurul A. Emran ${ }^{3}$, Zakiah Ayop ${ }^{4}$, Syarulnaziah Anawar ${ }^{5}$ \\ Centre for Advanced Computing (C-ACT), Faculty of Information and Communication Technology \\ Universiti Teknikal Malaysia Melaka, Hang Tuah Jaya, 76100 Durian Tunggal, Melaka, Malaysia
}

\begin{abstract}
This paper focuses on development of Smart Book Reader will help the blind people or who have low vision to read the book without using braille. This project utilises IoT technology with the use of an IoT device, IoT infrastructure and service. An IoT device, Raspberry $\mathrm{Pi}$, is used which is very energy efficient because it only uses $5 \mathrm{~V}$ of power to run. It is also a high portability device with only credit card size and can be carried out anywhere. Book reader will capture the picture of book pages using camera and book reader will process the images using Optical Character Recognition software. When the image is recognised, book reader will read it aloud ${ }^{1}$. Therefore, the blind people or those who have low vision will hear it without needing to touch using their fingertips. By using this book reader, the user can enjoy both softcopy and hardcopy books, by using online text to voice converter with a help of IoT connectivity protocol such as Wifi and 4G services. For hardcopy book, a camera is embedded to capture the page. The motivation to develop this product is to encourage all blind people to read ordinary books. This will help them to gain particular knowledge from the reading without a need to learn Braille.
\end{abstract}

Keywords-Internet of Things; Raspberry Pi; image processing; wellness; IR4.0; smart book reader

\section{INTRODUCTION}

There are approximately 285 million blind and visually problem people around the world. The term visual impairment covers a wide range and variety of vision, from lack of usable sight and blind, to low vision. Visually impairment cannot be corrected with eyeglasses or contact lenses to moderate visual impairment and an ability to read books, newspapers or any written notes. Visually impairment individuals usually only can read using the Braille system. The Braille system contains 63 codes of character. Each of them made of 1 to 6 raised dots in different position matrix or cells. Braille characters are embossed in lines on paper, and read by having the fingers passed lightly over the manuscript. The Braille system was invented by Louis Braille in 1824. Braille can be difficult to learn, not all people's fingertips are sensitive enough to use it. Furthermore, there are limitations to get books using Braille in the market [1].

Study in [2] shows that blind people face three aspects of difficulties in their daily life; environmental aspect, social aspect and technology aspect. For the environmental aspect, blind people often have difficulties in self-navigating outside well-known environments. Blind people also may face great difficulty when travelling or walking in a crowded place.
Because of this, they need to bring along well-sighted friends or family to help them. They usually learn every detail in their home environment. The obstacles such as table and chair must be placed in one location to prevent any undesirable events. In terms of the social aspect, blindness affects the person's ability to complete the job duties. Because of this, job opportunities for blind people are limited. This will affect their finances as well as their self-esteem. In the technology aspect, blind people cannot read an information on a web page. Blind people also have difficulties to use devices that require visual selection such as music player.

On the other hand, Internet-of-Things (IoT) has been referred to as an important keyword in shaping the future to support human life. It is because of its capability to ensure connectivity between people and their machines to support data reachability, so that people from anywhere using an existing Internet service such as cloud can reach the data, which is automatically collected by the devices/machines. The IoT can be defined as the network of physical objects that contains embedded technology to communicate and sense or interact with their external states or environment [3]. IoT refers to millions of devices that are connected to the Internet, sharing data and collecting data. The input device such as sensors and the single board computer such as Raspberry $\mathrm{Pi}$ will be integrated together to create the IoT device. There are a plenty of sensors for use such as the PIR sensor, ultrasonic sensor, soil moisture sensor and many others. Besides sensors, another input device such as a camera can also be used in IoT applications. Cameras can collect visual data used in any IoT application such as surveillance systems and detection systems [4]-[9]. Once the data from IoT device is collected, it will be sent through IoT network connectivity such as WiFi and LTE to the user.

This project is designed to overcome Braille problem using IoT technology. This Project is built using a small size and low cost single board computer, named Raspberry Pi. Raspberry Pi that has been introduced by Eben Upton, where it is a cheap but with a high mobility microprocessor is one example of high potential IOT device, where it enables a Machine-to-Machine Communication using IEEE 802 standard [10]. The camera acts as an input device where visual data is collected. The visual data is sent to the single board computer using WiFi connection. The image is processed to perform image to text conversion and text to voice conversion using available converters from the online site.

${ }^{*}$ Corresponding Author 
The developed book reader will help the blind people or those who have low vision to read the book without using braille. Book reader will capture the picture of book pages using a camera and then process the images using OCR software. When the image is recognized, book reader will read it aloud. Thus, the blind people or those who have low vision will hear it without the need to touch using their fingertips.

The developed Smart Book Reader will help blind and visually impairment people in reading. This reader will help to reduce the weakness of the braille. Braille is a system of raised dots that can be read with the fingers by people who are blind or who have low vision. Braille can be difficult to learn, not all people's fingertips are sensitive enough to use it. Furthermore, there are limitations to get book using braille in market. By using this book reader, most of blind and visually impairment people can enjoy various books as much as ordinary people, without concerning braille system. Book Reader will read aloud a book without need to touch like braille

This paper is organized as follows. Section 2 describes the prototype design used to develop the IoT based security system. Section 3 introduces implementation stage used throughout this paper. The description of testing stage and discussion is shown in Section 3, followed by conclusion in Section 4.

\section{Methodology}

Rapid Application Development model is applied to develop the system as shown in Fig. 1. The development process goes through the requirements planning phase, user design phase, construction phase and cutover phase.

- Requirements Planning Phase: In this phase, we analyze problems that occurs among blind people when reading a book, and then determine adequate solutions that might solve the problems. We also identified hardware and software required for the development.

- User Design Phase: In this phase, the problems that occur among visual impairment person is analyzed to determine adequate solution/modules that might help them and their family in having a low cost, portable and easy to use product. The hardware and software required for the development are also identified in this phase.

- Development Phase: In this phase, the system based on design in the user design phase is developed. Early tests to ensure functionality of the system have been done.

- Cutover Phase: In this phase, the functionality of the system is improved based on testing in the previous stage. The overall tests for the developed system are then finalized.

\section{A. Prototype Design}

Fig. 2 shows the design of the developed Book Reader consists of Raspberry Pi, Pi Camera and a stand.
The physical design of the prototype is shown in Fig. 3. The Raspberry Pi is integrated with Pi camera for capturing book pages that will be converted to text. The text will be sent to text to audio converter, where the speaker installed in the Raspberry Pi will play the audio. Tesseract and Flite software are used to implement image-to-text conversion and text-tovoice conversion. The software can be accessed through internet using IEEE802.11 standard connectivity, embedded in Raspberry Pi.

Fig. 4 shows the flowchart for the Book Reader system using Raspberry Pi. When the Book Reader is activated, the Pi Camera will capture the image. When the image is captured, the image will be converted into text file. If not, the camera will capture the image again. After that the text file will be read aloud as sound.

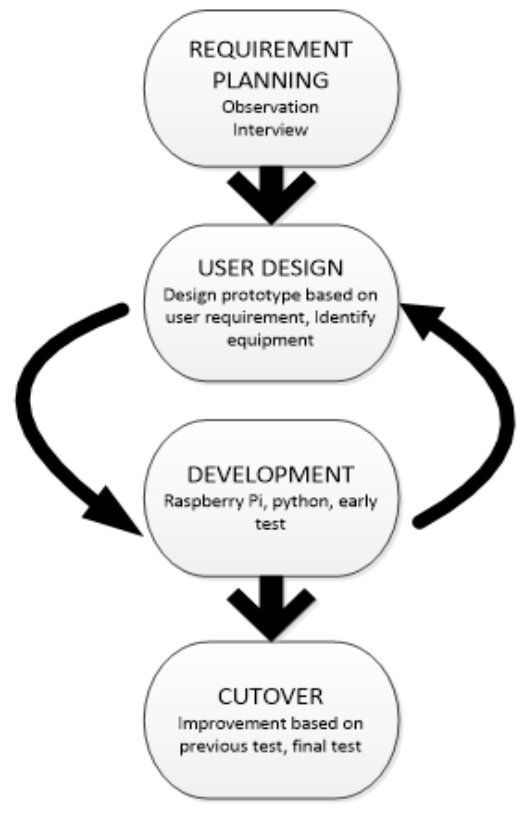

Fig. 1. Rapid Application Development Phase

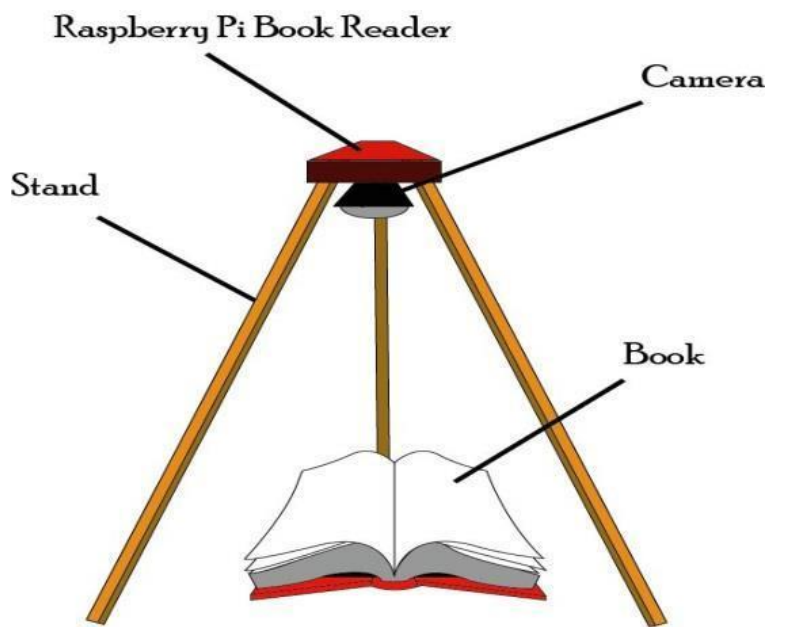

Fig. 2. Physical Design of Developed Book Reader using Raspberry Pi. 


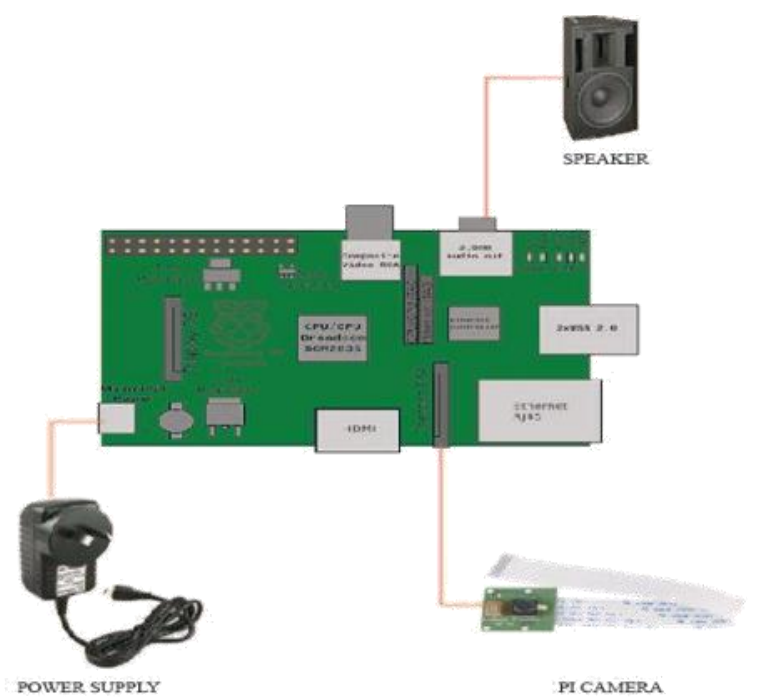

Fig. 3. Physical Design of the developed Book Reader.

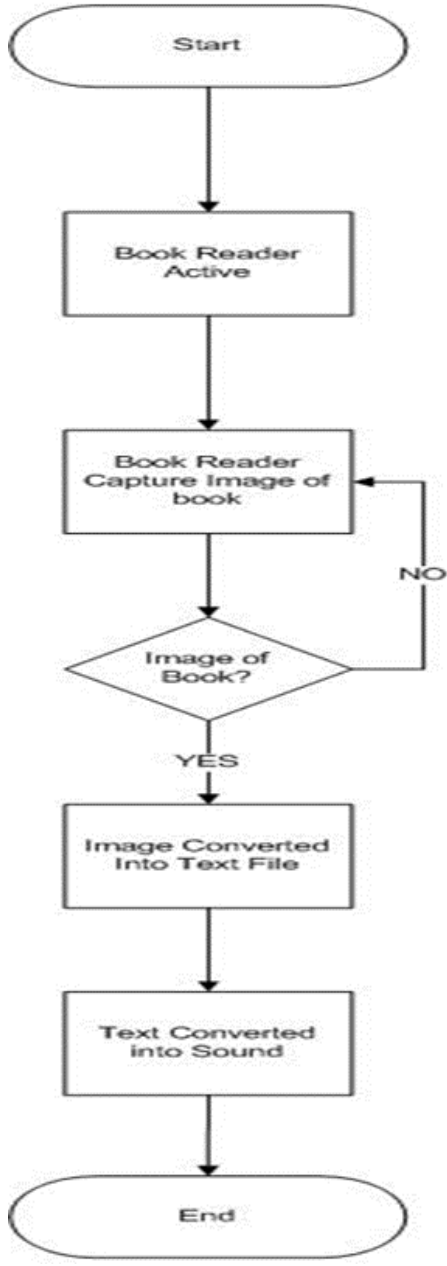

Fig. 4. Flow Chart for Book Reader using Raspberry Pi Project.

\section{B. Implementation}

This stage consists of three steps; hardware setup, software setup and book reader setup. The required software and hardware are shown in Table 1.
TABLE I. HARDWARE AND SOFTWARE REQUIREMENT FOR THE DEVELOPED PROTOTYPE

\begin{tabular}{|c|c|}
\hline Hardware & Software \\
\hline $\begin{array}{ll} & \text { Raspberry Pi } 2 \\
\text { - } & \text { Micro SD Class } 10 \\
\text { - } & \text { USB Mouse } \\
\text { - } & \text { USB Keyboard } \\
\text { - } & \text { Monitor with HDMI } \\
\text { - } & \text { Power Supply } \\
\text { - } & \text { Pi Camera } \\
\text { - } & \text { Stand } \\
& \text { Speaker }\end{array}$ & $\begin{array}{ll} & \text { OS for } \\
- & \text { Raspberry pi (Noobs) } \\
\text { - } & \text { SD Formatter 4.0 USB } \\
\text { - } & \text { Tesseract } \\
\text { - } & \text { Flite }\end{array}$ \\
\hline
\end{tabular}

Fig. 5 and 6 show hardware setup for the developed book reader. The hardware listed in Table 1 is setup for development purpose only. The finalized prototype only consists of Raspberry Pi embedded with camera, SD card, Wi-Fi dongle and power supply/power bank as illustrated in Fig. 6 .

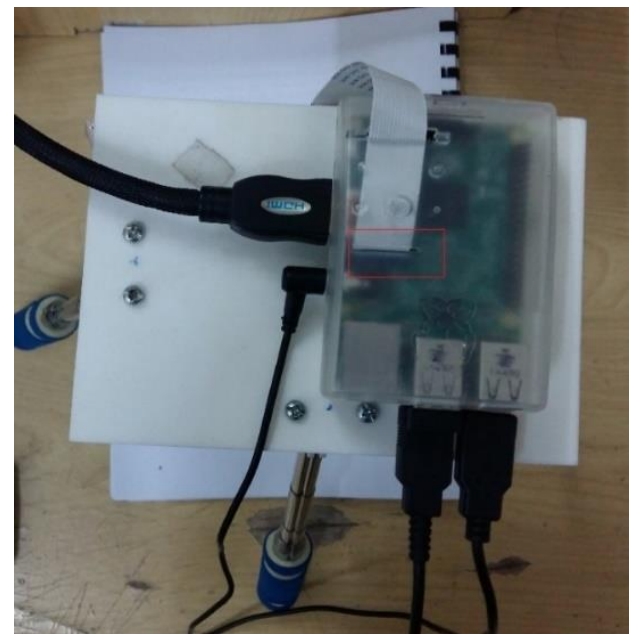

Fig. 5. Hardware Setup for Development Purpose.

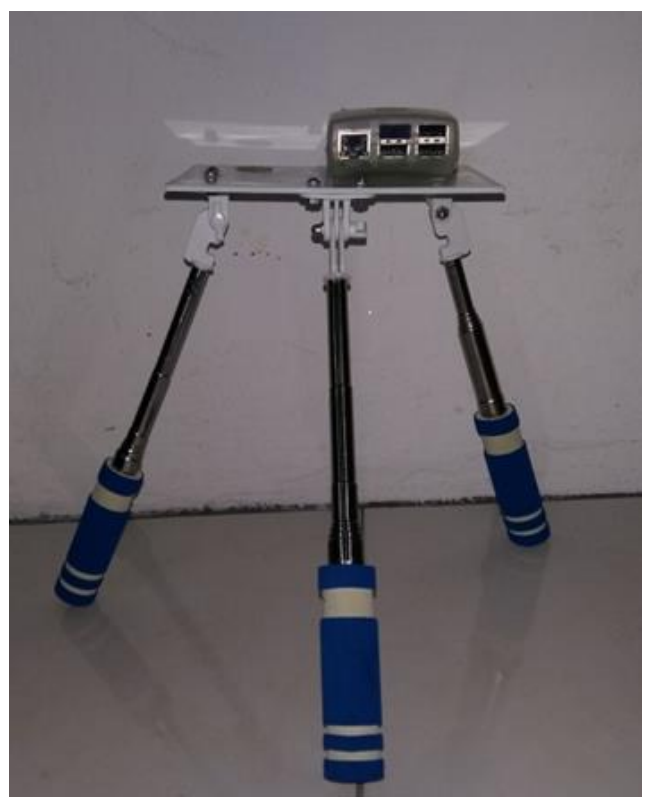

Fig. 6. Developed Prototype. 


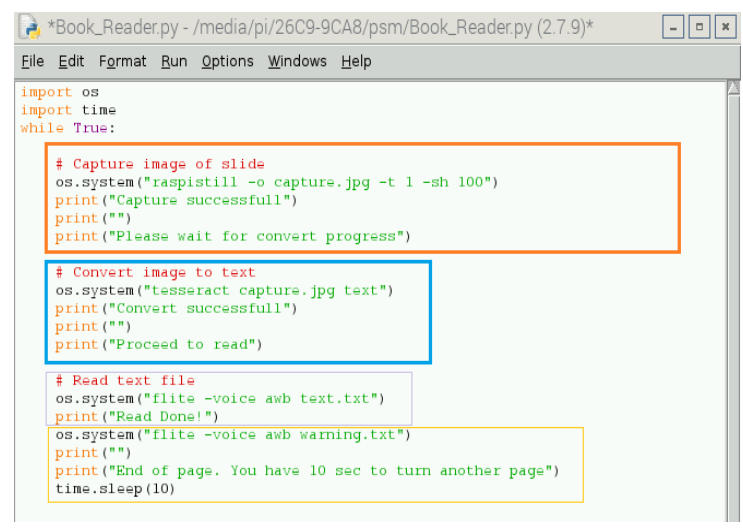

Fig. 7. Book Reader Configuration.

For book reader setup, python programming has been used as shown in Fig. 7. The embedded camera is used to capture the image of the book. The captured image is sent to Tesseract that recognizes the word in the image and converts it into text file. Then Flite will read the text file and the text file will be converted to voice and played by speaker. The Python code is also developed for Flite to read warning.txt as shown in Fig. 8 to ask the blind people to turn the next page. In this prototype, 10 second is set to give time to blind people turning page.

\section{TESTING AND DISCUSSION}

This chapter discusses about testing methods of the project. The testing phase consists of three types of testing; the testing of camera functionality, the sound functionality, image-to-text conversion, and text-to-sound conversion testing.

For camera functionality test, the test to ensure the Pi camera can properly capture and save the captured image in a correct folder has been done and shown in Fig. 8. The captured image will be sent to the image text converter in the next stage.

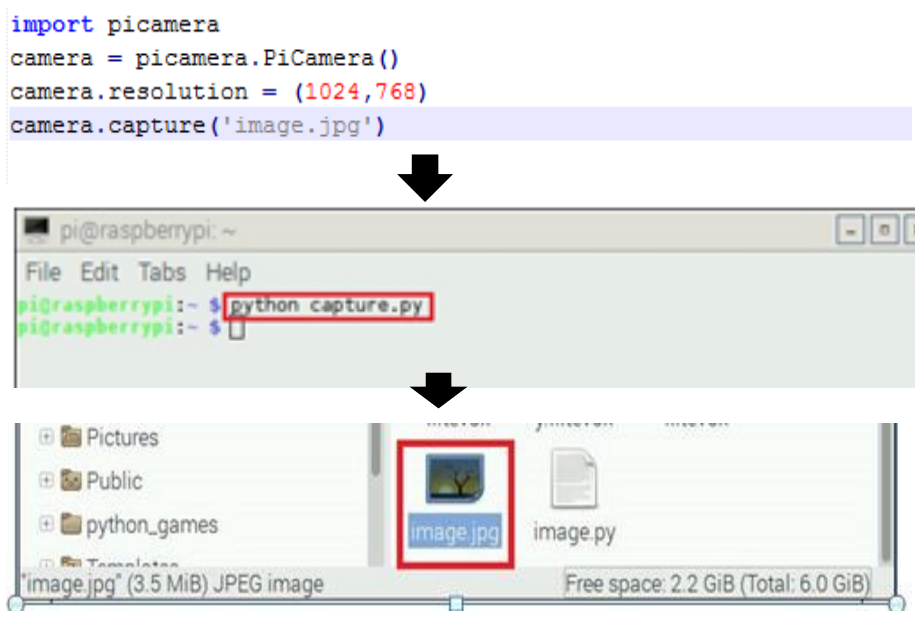

Fig. 8. Camera Functionality Test.
The next testing is image-to-text conversion test, shown in Fig. 10. Tesseract software has been used to implement imageto-text conversion. The Tesseract-OCR software has been tested by using an image of a book that has been captured and saved in a particular folder. Fig. 10 shows the command to run the Tesseract and the text file that have been converted from the image file in the folder /home/pi.

The final step of testing is to ensure the functionality of text-to-sound conversion. Flite software has been used. The testing command line and the result are shown in Fig. 11.

The test to ensure the functionality of the speaker to play the sound has also been done and shown in Fig. 9. This test is essential for use in the text to voice testing part.

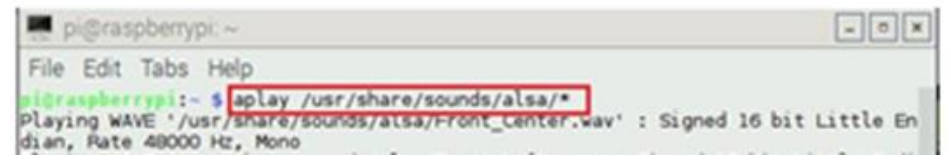

Fig. 9. Sound and Speaker Test.

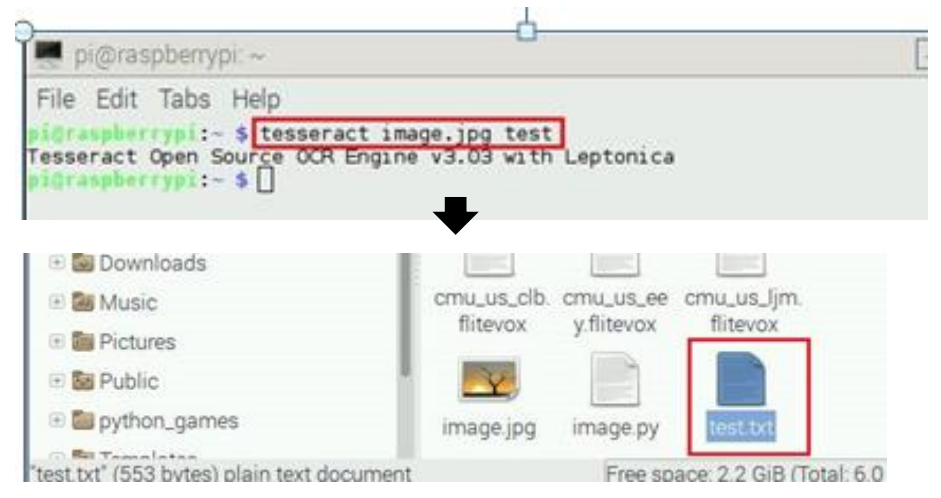

Fig. 10. Image-to-Text Conversion Testing using Tesseract.

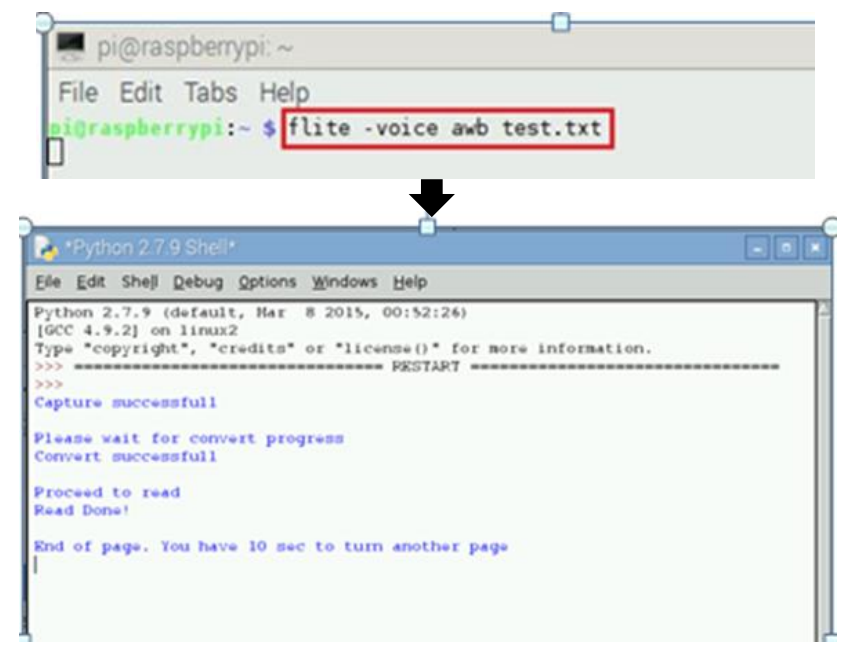

Fig. 11. Text-to-Sound Conversion using Flite Software. 


\section{CONCLUSION}

In this paper, the development of Book Reader for Blind People has been discussed. The Book Reader is developed using an IoT device; Raspberry Pi, which is low on power consumption, and being small in size that contributes to a high portability device for blind people. The product also can be realized with the help of IoT supporting network protocol such as $\mathrm{WiFi}$ and $4 \mathrm{G}$. By using this book reader, most of the blind and visually impaired people can enjoy various books just as much as ordinary people, without being concerned with the Braille system. Book Reader will read aloud a book without the need for touch like Braille.

\section{ACKNOWLEDGMENT}

This paper is funded by Global Commission on the Stability of Cyberspace (GCSC) Grant (GLUAR/HGCC/2018/FTMK-CACT/A00015). A high appreciation goes to Fakulti Teknologi Maklumat dan Komunikasi (FTMK), Universiti Teknikal Malaysia Melaka (UTeM) for facilitating the work done in this paper.

REFERENCES

[1] http://www.wipo.int/pressroom/en/briefs/limitations.html
[2] http://www.livestrong.com/article/241936-challenges-that-blind-peopleface/

[3] Patel, Keyur K., Sunil M. Patel, and PG Scholar1 Assistant Professor. "Internet of Things-IOT: definition, characteristics, architecture, enabling technologies, application \& future challenges." International Journal of Engineering Science and Computing 6, no. 5 (2016).

[4] Andreas P. P., Kostas E. P., Yutaka I. and Byung G. K. ," IoT-based surveillance system for ubiquitous healthcare," IIECON 2016 - 42nd Annual Conference of the IEEE, 23-26 December 2016.

[5] M. Kochlan, "Wireless Sensor Network for Traffic Monitoring using Raspberry Pi Board" Proceedings of the 2014 Federated Conference onComputer Science and Information Systems pp. 1023-1026.

[6] L. Ada, PIR Motion Sensor [Online]. Available: FTP: https://learn.adafruit.com/pir-passive-infrared-proximity motionsensor/overview

[7] N. Yang, "Motion Sensor and Camera Placement Design for In-home Wireless Video Monitoring Systems", IEEE Globecom 2011, , 5-9 Dec, Texas, USA.

[8] S. V. Gawande and P. R. Deshmukh, "Raspberry Pi Technology," International Journal of Advanced Research in Compute Science and Software Engineering, Vol.5, No.4, April 2015.

[9] D. Aishwarya and J. A. Renjith, "Enhanced Home Security Using IOT and Raspberry Pi," International Research Journal of Engineering and Technology, Vol. 4, No.4 April 2017.

[10] C. Severence, "E. Upton:Raspberry Pi," IEEE Computer Magazine, Vol. 46, Issue. 10, pp.14-16, 2013. 